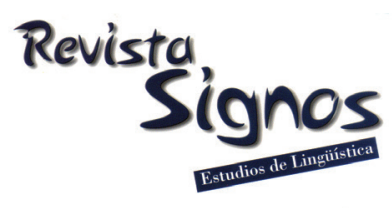

\title{
Sobre la categorización de seem en inglés y su traducción en español. Análisis de un corpus paralelo ${ }^{1}$
}

\author{
On the categorization of seem and its Spanish renderings. \\ Analysis of a parallel corpus
}

\author{
Francisco Alonso Almeida \\ Universidad de Las Palmas de Gran Canaria \\ ESPAÑA \\ falonso@dfm.ulpgc.es
}

\author{
María Luisa Carrió Pastor \\ Universitat Politècnica de València \\ ESPAÑA \\ lcarrio@idm.upv.es
}

Recibido: 8-III-2013 / Aceptado: 5-XI-2014

\section{Resumen}

El objetivo principal de este trabajo es categorizar los valores de seem en un corpus paralelo de textos técnicos en lengua inglesa y española. Atendiendo a cuestiones sintácticas y pragmáticas, se establecen varias categorías de esta forma verbal, lo que incluye la división de seem en mecanismos evidenciales y epistémicos. Los datos se han tomado del Corpus of English-Spanish software localization de la Universidad de Vigo (LOGALIZA), el cual es una compilación de textos técnicos en lengua inglesa con su traducción al español. Los resultados de nuestros análisis reflejan que la traducción de seem depende, en gran medida, de la reflexión que el traductor hace de esta forma durante el proceso traductológico pero, en general, se concluye que se hace un uso evidencial de la forma seem en un porcentaje alto de casos.

Palabras Clave: Seem, evidencialidad, modalidad, traducción. 


\begin{abstract}
The main objective of this paper is to categorize the values of seem in a parallel corpus of technical texts in English and Spanish. Considering syntactic and pragmatic issues, we offer a taxonomy of seem forms. This also includes the classification of this verb as an evidential or as an epistemic device. Data for analysis were taken from the Corpus of English-Spanish software localization at the University of Vigo (LOGALIZA), which is a compilation of technical texts in English with their corresponding translations into Spanish. The results of our analyses show that the translation of seem largely depends on the translator's own assumptions concerning the meaning of this form in the original texts during the process of translation. We conclude that seem has an evidential function in a high percentage of the analyzed samples.
\end{abstract}

Key Words: Seem, evidentiality, modality, translation.

\title{
INTRODUCCIÓN
}

La forma verbal seem refleja una perspectivización del hablante con respecto a la información que se presenta. En este sentido, seem puede tener diferentes valores semánticos y pragmáticos dependiendo del contexto en el que se inserta, además de la posible intención del hablante y las interpretaciones que se haga del mismo. Así, seem puede considerarse como evidencial, esto es, comunica la fuente de información o el modo en el que esta se obtiene (las inferencias, por ejemplo). Muchos autores también lo categorizan como un hedge $e^{2}$ Hyland, 1998) con valor epistémico, que evalúa la proposición en términos de verdad y certeza, lo que se traduce en varios grados que reflejan el compromiso del autor con su texto, sin embargo, en otras ocasiones, seem funciona claramente con valor copulativo.

El concepto de evidencialidad es considerado con frecuencia como parte del dominio epistémico, ya que la indicación de la fuente de información implica indefectiblemente el compromiso del autor con la veracidad de lo expresado para algunos autores (Chafe, 1986; Palmer, 1986; Mushin, 2001) lo que, a su vez, refiere a grados de probabilidad de que una determinada proposición sea cierta. Sin embargo, otros especialistas sostienen que los mecanismos evidenciales no reflejan necesariamente valores de probabilidad y consideran la evidencialidad y la modalidad epistémica como conceptos diferenciados (Marín Arrese, 2009), aún cuando se produzca superposición funcional, como explicaremos más adelante.

Estas ideas sobre la relación que existe entre evidencialidad y modalidad epistémica dan lugar a metodologías diferentes tal y como apuntan Dendale y Tasmowski (2001) que las categorizan como inclusión, intersección y disyunción. Como primera aproximación, tomaremos el ejemplo (1):

(1) He escuchado que la conferencia ha sido un éxito. 
En este caso, siguiendo una perspectiva inclusiva, el uso de la matriz 'he escuchado que' implica exclusivamente el grado de posibilidad de que la información que se ofrece en la cláusula subordinada sea cierta. En el caso del modelo intersectivo, se indica tanto la fuente de información, auditiva en este caso, como la evaluación que se hace de la información aportada en la cláusula 'la conferencia ha sido un éxito' según la perspectiva del hablante indicada en la matriz. En definitiva, tanto la inclusión como la disyunción, presentan una interpretación del mensaje en términos de verdad, certeza y probabilidad. Sin embargo, el modelo disyuntivo establecería que la matriz evidencial solo indica de dónde se ha obtenido la información.

En el caso de 'he escuchado', haciendo una interpretación literal, se trata de información que se ha oído a terceros. En este modelo, no se cuestiona la veracidad de la información como sí ocurre en los dos modelos explicados anteriormente. En nuestro trabajo, seguimos este modelo disyuntivo sin perjuicio de que se deriven otros efectos pragmáticos del uso de los mecanismos evidenciales. Según este modelo, la evidencialidad se refiere exclusivamente a la manera en la que se obtiene la información o la referencia a la fuente de la misma sin relación semántica con la modalidad epistémica, por lo que los mecanismos evidenciales no implican grado de probabilidad o posibilidad.

En este artículo mostraremos los resultados del análisis de seem realizado en un corpus paralelo de textos no literarios extraído mediante el uso de una herramienta informática que nos ofrece los ejemplos de seem en la lengua original (inglés), así como su valor en la lengua meta (español), con el fin de clasificar los valores semánticos que adquiere seem y su implicación en el debate que existe sobre los conceptos de evidencialidad y modalidad epistémica. Para ello, determinamos primero qué entendemos por unidad evidencial y si esta comprende solo estructuras gramaticales, como apunta Aikhenvald (2004), o también se pueden incluir estructuras léxicas o incluso estructuras complejas, en el sentido de Pietrandrea y Stathi (2010), que describiremos más adelante. Este estudio contribuye en parte a la creación de un catálogo de valores de seem, con especial atención a su categorización como evidencial, tanto en la lengua origen como en la lengua meta.

En principio, estos valores pueden ser epistémicos o evidenciales desde una perspectiva semántica, aunque seem también puede usarse como verbo copulativo desde una perspectiva sintáctica pero, en este caso, no indicaría probabilidad, esto es valor epistémico, ni evidencialidad, esto es modo de adquirir la información, como se muestra en la sección 3.2. de este trabajo. La manera en la que se traslada el sentido de uso de esta forma verbal al español indica la percepción del traductor con respecto al uso que seem presenta en la lengua origen. Siguiendo a Wierzbicka (2006), quien apunta que el uso de esta forma verbal obedece a una opción personal del hablante frente a otras expresiones similares para la autora como I presume, nuestra hipótesis inicial es que la traducción de seem en español tiende a reflejar esta 'opción personal' frente a otras opciones translativas válidas lo que se puede reflejar en la 
propia configuración sintáctica en la lengua meta que preserva la sintaxis original. Dicho esto, nuestro objetivo principal es categorizar los valores de seem en nuestro corpus paralelo de textos técnicos en inglés y español, teniendo en cuenta cuestiones sintácticas y pragmáticas en la interpretación de los ejemplos analizados que, como veremos, difieren del significado puramente epistémico que Wierzbicka (2006) interpreta en el uso de seem en matrices como It seems (that) y It seems to me, siendo más destacado el uso evidencial de esta forma.

El contenido de este trabajo se ha dividido en varias secciones con el fin de indicar los pasos seguidos en el análisis. En la sección 1, marco teórico, se explican primero los significados de seem según el Oxford Dictionary English (OED, 2013); a continuación, se ofrece una descripción de los conceptos de modalidad epistémica y evidencialidad y la manera en la que éstos se relacionan según los posicionamientos teóricos mencionados anteriormente. Seguidamente, se presenta el corpus de trabajo, así como el método de análisis en la sección 2, marco metodológico. Los resultados y la discusión de los mismos se incluyen en la sección 3 y, finalmente, se presentan las conclusiones que se derivan de este estudio junto con las sugerencias para futuras investigaciones sobre el tema.

\section{Marco teórico}

\subsection{El significado del verbo seem}

Entre los estudios que versan sobre el verbo seem, Aijmer (2009) presenta una de las aportaciones más destacadas en este campo pues ofrece un estudio minucioso de la traducción de seem al sueco y sus implicaciones. De esta manera, Aijmer (2009) muestra que el análisis de los procesos de traducción de seem permite la caracterización de este verbo, ya que la traducción tiende a reflejar la manera en la que esta forma se interpreta por el receptor. Seem es un verbo de percepción en inglés, como see, sound y feel, pero su significado es mucho más 'impreciso' según Aijmer (2009) y, en ocasiones, se asemeja a verbos de tipo cognitivo como think.

El Oxford Dictionary English define este verbo con las entradas 1, 2, 3, 4, 4f, 9a y $9 \mathrm{~b}$, que citamos a continuación. A estas definiciones hemos adjuntado los ejemplos (2), (3), (4), (5), (6) y (7), tomados de la misma fuente, aunque hemos modernizado la letra thorn usando th en su lugar. La traducción al español que se muestra ha sido realizada por los autores de este estudio, excepto en los ejemplos (8) y (9), en los que incluimos las traducciones de Cohen (en Jonson, 1983) y Martín Lorente (en Pepys, 2007), respectivamente:

1. To be suitable to, befit, beseem. Often with adv., well, best, fair, etc.

(2) It semes a kynge to haue discrescioun (c1400 Secreta Secret.); 'Es conveniente que un rey muestre discreción'.

2. refl. and intr. To vouchsafe, deign. 
(3) I prai leuedi if thou wald seme, To tak this littel werc to quem (a1300 Cursor Mundi); 'Te ruego, señora, si lo consideras oportuno, que aceptes este pequeño trabajo'.

3. With n., adj., or phr. as compl.: to appear to be, to be apparently.

(4) A silly rogue, but one that would seem a gentleman (1667 S. PEPYS DiarY); 'Un pícaro tonto pero que parece un caballero'.

4. With infinitive: To appear to be or to do something.

(5) Fair creature! Thou dost seem to be Some wandering spirit of the sea (1812 J. WILSON ISLE OF PALMS); ‘¡Pobre criatura! Parece que eres un espíritu errante del mar'.

4. f. To appear to oneself; to image oneself, or think one perceives oneself, to (do something).

(6) Somehow I can't seem to get warm (1969 M. Pugh LAST PLACE LeFT); 'De alguna manera parece que no puedo entrar en calor'.

9. a. trans. To think, deem, imagine.

(7) They can because they seeme they can (1627 G. Hakewill APOL.); 'Pueden porque parece que pueden'.

9. b. To think fit. Obs.

(8) And Beneath your threshold, bury me a Loade-stone To draw in Gallants, that weare spurres: The rest, They'll seeme to follow (1612 B. Jonson Alchemist); 'Y debajo del umbral, entiérrame una piedra imantada para atraer a los valientes con espuelas, el resto vendrá solo’ (traducción de Cohen, 1983: 56).

A estas acepciones que aparecen en el OED, Nakamura (2010) ofrece otra adicional, de acuerdo al corpus que propone para su estudio, con el significado de 'fingir': "To feign an action, character or the like; make oneself seem (to do or to be); assume an air; pretend. Obs. rare. exc. dial.? Now U.S.” (Nakamura, 2010: 231). Este significado, que se ejemplifica en (9), estuvo vigente en el inglés británico hasta 1900 y, según apunta Nakamura (2010), ahora solo se encuentra en dialectos y en el inglés americano.

(9) My wife this evening discoursing of making clothes for the country; which I seem against, pleading lack of money, but I am glad of it in some respects (1663 Pepys, Diary, IV 158 in Nakamura, 2010: 226) 'Esta noche, mi esposa me habló de hacerse ropa para el campo, pero me mostré en contra alegando falta de dinero; sin embargo me alegro' (traducción de Martínez Lorente, 2007: 274).

Hemos de apuntar que en el corpus de este estudio no hemos encontrado evidencias de la traducción de seem con el significado 'fingir'; no se han hallado ni en el corpus de textos originales, ni en el corpus de textos meta. 


\subsection{Modalidad epistémica y evidencialidad}

El estudio de seem requiere que, inevitablemente, se describan los conceptos de modalidad epistémica y evidencialidad, así como la relación que existe entre ambos. Por lo general, seem se ha considerado como una forma verbal cuya función es tanto la de mostrar la fuente y/o el modo de la información como la de expresar falta de seguridad o compromiso con la información que se presenta, además de expresar un valor perceptual, como señala Usoniené (2000). Estos valores dependen de los complementos que seem tenga, según explica esta autora:

"Only structure with full infinitival forms can be regarded as conveying information that is modal, because they indicate the speaker's assessment of the proposition asserted in terms of greater/lesser degree of certainty. By contrast, structures with zerocomplementation are considered non-modal, because they report on the evaluatively charged qualitative characteristic of some perceptual stimulus" (Usonienè, 2000: 203).

En nuestro trabajo, mostraremos que el valor modal de seem depende en gran medida del componente sintáctico, como sucede en el caso del verbo seem seguido de predicativo, pero además el componente pragmático es fundamental para determinar el uso concreto de esta forma.

En este contexto, el concepto de evidencialidad se define como la codificación de la fuente o el modo de adquirir dicha información. Para muchos, la expresión de la fuente implica grados de seguridad del autor con respecto a la proposición que se expresa, esto es, modalidad epistémica. Nuyts (2001: 21) define la modalidad epistémica de la siguiente manera:

"[...] the evaluation of chances that a certain hypothetical state of affairs under consideration (or some aspects of it) will occur, is occurring or has occurred in a possible world".

Según la manera de relacionar estos conceptos, se producen tres posibles propuestas metodológicas, como ya se mencionó en la introducción, que son inclusión, intersección y disyunción. En el caso de la primera, la evidencialidad se integra como subdominio de la modalidad epistémica (Palmer, 1986), tal y como se muestra en (Kranich, 2009: 28):

"Furthermore, the evidential use of modals in English is always also epistemic, in that the speaker does not express absolute certainty as to the truth of the proposition, but only that, on the grounds of certain evidence, the proposition has a certain likelihood of being true. The use of evidential markers which provide information as to the source of the evidence but do not also cast a shade of doubt on the truth of the proposition is probably rather evidenced in languages where evidential modality is a more clearly grammaticalized category than in English, and where evidential markers also convey for example that the proposition refers to something personally witnessed by the speaker." 
En cuanto a la segunda, la evidencialidad y la modalidad epistémica son dominios diferentes, pero tan relacionados que se considera que la expresión de la fuente de información indica el compromiso del hablante con la proposición expresada, entre otros aspectos. En este sentido, Diewald, Kresic y Smirnova (2009: 190) indican:

"Evidentiality is concerned with indicating the information source the speaker is relying on to make a claim. This places this category next to epistemic modality without, however, merging them into one".

En estos dos posicionamientos el grado de certeza está directamente relacionado con el tipo de evidencia. La evidencia puede ser directa e indirecta según apunta Willet (1988). Ferrari (2006) denomina la evidencialidad directa con el nombre de evidencialidad sensorial y a la indirecta como citativa. La categorización de las evidencias se resume en la Figura 1:

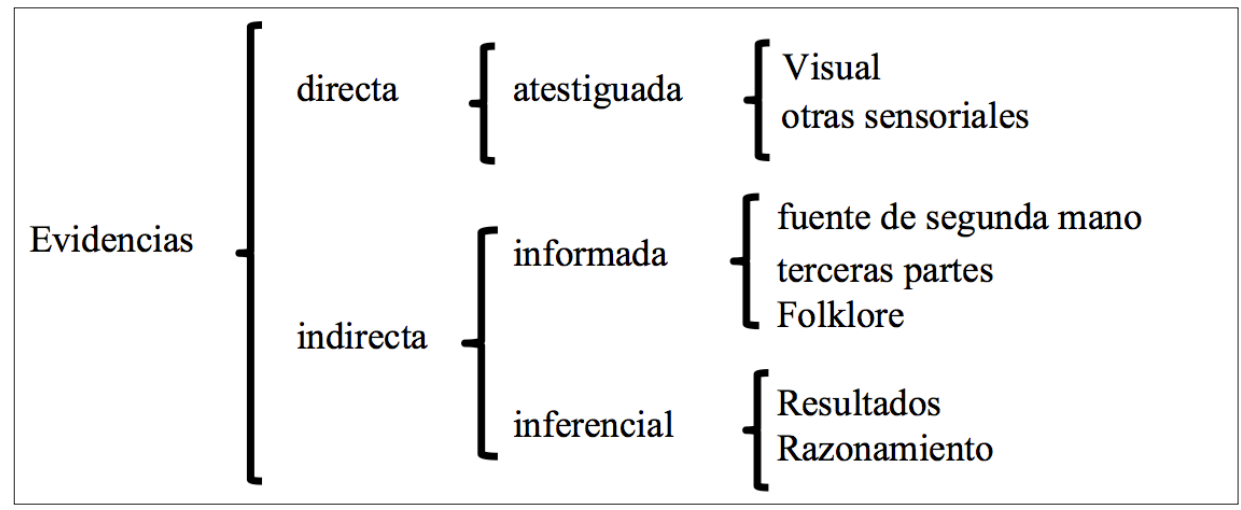

Figura 1. Categorías de evidencias (Willet, 1988: 57).

De acuerdo con esta clasificación, el grado de certeza estaría determinado por la naturaleza de la evidencia. Se considera que las evidencias directas indican que se tiene constancia fehaciente sobre la veracidad mientras que, en el caso de las evidencias indirectas, el grado de certeza es menor puesto que no se tiene contacto visual o auditivo. En este contexto, se entiende que los mecanismos evidenciales se considerarían hedges que, con frecuencia, se dice que indican falta de compromiso del hablante con la información expresada (Hyland, 1998; Alonso Almeida, 2012).

Finalmente, la relación disyuntiva significa que los conceptos representan dominios diferentes; por lo tanto, la expresión de las evidencias para expresar una determinada proposición no indica necesariamente valores de verdad proposicional, compromiso de los autores o falta de certeza con respecto a la información que proporcionan, como apunta Cornillie (2009: 59):

"Reliability should not be lumped together with the evaluation of likelihood and its expression in terms of degrees of epistemic 
speaker commitment. Whereas reliability is included in epistemic modality, the reverse is not true. The reliability of the assessment of likelihood is measured in terms of degrees of speaker commitment. By contrast, the reliability of evidential values cannot be presented in terms of an assessment of likelihood. Yet, this is exactly what many accounts do. A further difference between reliability and likelihood consists in the fact that the former notion mainly refers to states of affairs that have happened or, at least, are happening, whereas the latter also refers to the future."

En nuestro análisis, siguiendo las indicaciones de Cornillie (2009), la evidencialidad y la modalidad epistémica se consideran dos dominios independientes. Esto significa que la evidencialidad no guarda necesariamente relación con la expresión de compromiso, de seguridad o de certeza por lo que los evidenciales no se incluirían como un tipo de hedges. Así, explicaremos que seem y su traducción al español, 'parecer', presentan un significado primario evidencial, a excepción de su uso copulativo que parte de un principio de comparación, como en el ejemplo 'La casa parece nueva' en el que su significado está más próximo a la forma 'estar' en español.

\section{Marco metodológico}

\subsection{Descripción del corpus}

Los datos para el análisis pertenecen al Corpus of English-Spanish software localization de la Universidad de Vigo (LOGALIZA), que es una compilación de textos paralelos en inglés y su traducción en español. El corpus contiene un total de 4.992 .133 palabras que se distribuyen en 2.342.099 palabras en el caso del sub-corpus de inglés y 2.650.035 en el caso del sub-corpus de español. Esta cantidad se reparte en un total de 226.500 unidades de traducción. La persona encargada de coordinar la realización de este corpus es Mercedes Rodríguez García. LOGALIZA se encuentra en red y dispone de una interfaz que permite hacer búsquedas siguiendo una sintaxis predeterminada de acuerdo con PCRE (Perl Compatible Regular Expressions), tal y como se apunta en la página de ayuda a la búsqueda de este corpus (http://sli.uvigo.es/CLUVI/axuda_ en.html).

\subsection{Método de trabajo}

Para nuestro propósito, hicimos la búsqueda informatizada de la cadena seem $^{*}$ en el corpus referido, donde el asterisco representa todos aquellos caracteres que no se especifican. Esta búsqueda devolvió todas las variantes de esta forma verbal: seem, seems, seemed, etc. Una vez obtenidos estos datos, se clasificaron atendiendo a su forma en lengua inglesa en verbos seguidos de to, matrices con that y con as if, y las variantes copulativas de seem, siguiendo parcialmente el modelo propuesto por Aijmer (2009).

Aijmer (2009) categoriza los ejemplos de seem de acuerdo con su organización sintáctica, en los que puede estar acompañado por experimentadores o no. Esto 
se observa en el ejemplo 'She seems to have solved the puzzle' y en 'It seems that it won't rain today'. La enorme variación sintáctica de esta forma verbal ha sido descrita por Johansson (2007), quien agrupa los ejemplos de seem en (a) seem copulativo (seem + adjective), (b) seem con complemento clausal (seem that), y (c) verbo catenativo (seem to + infinitive). Volviendo a la clasificación de Aijmer (2009), esta es mucho más detallada (los ejemplos que se citan a continuación son de la misma fuente): (a) seem + that-clause (It seems that it is raining), (b) seem + as if (like) (It seems as if it is raining), (c) seem + like noun phrase (It seems like a fool), (d) seem + infinitive (It seems to be raining), (e) seem + adjective (participle) (He seems agitated), (f) seem + adjective + that-clause (It seems possible that it will be raining), $\mathrm{y}(\mathrm{g})$ parenthetical seem (It is raining it seems). De estas categorías, solo se han encontrado en este estudio ejemplos de (a), (b), (d) y (e), como describimos en el siguiente apartado en el que se muestran los resultados.

\section{Resultados y discusión de los datos}

Los resultados de nuestros análisis de las traducciones al español del verbo seem se muestran en porcentajes y en casos (entre paréntesis), como se observa en la Tabla 1. En la primera columna de la izquierda hemos incluido las combinaciones del verbo seem encontrados en el corpus. En las filas superiores aparecen las equivalencias encontradas en español, mientras que en la columna de la derecha aparece el total de casos de cada combinación de seem en inglés, teniendo en cuenta sus equivalencias en español. En la fila inferior aparecen los porcentajes y casos de las traducciones de seem en español:

Tabla 1. Frecuencia de las estructuras de seem en los textos originales y sus equivalencias en los textos meta.

\begin{tabular}{|l|c|l|c|c|c|r|}
\hline & $\begin{array}{l}\text { PARECER + } \\
\text { INFINITIVO }\end{array}$ & $\begin{array}{l}\text { PARECER } \\
\text { COPULATIVO }\end{array}$ & $\begin{array}{l}\text { PARECE } \\
\text { QUE }\end{array}$ & AUSENCIA & MODAL & TOTAL \\
\hline SEEM TO & $46,39 \%(77)$ & $4,22 \%(7)$ & $16,87 \%(28)$ & $12,65 \%(21)$ & $1,20 \%(2)$ & $81,33 \%(135)$ \\
\hline COPULAR SEEM & $0,60 \%(1)$ & $12,05 \%(20)$ & $0 \%(0)$ & $1,20 \%(2)$ & $0 \%(0)$ & $13,85 \%(23)$ \\
\hline IT SEEMS (THAT) & $0 \%(0)$ & $0 \%(0)$ & $4,82 \%(7)$ & $0 \%(0)$ & $0 \%(0)$ & $4,22 \%(7)$ \\
\hline IT SEEMS AS IF & $0 \%(0)$ & $0 \%(0)$ & $0,60 \%(1)$ & $0 \%(0)$ & $0 \%(0)$ & $0,60 \%(1)$ \\
\hline TOTAL & $46,99 \%(78)$ & $16,26 \%(27)$ & $21,68 \%(36)$ & $13,85 \%(23)$ & $1,20 \%(2)$ & $100 \%(166)$ \\
\hline
\end{tabular}

Como hemos comentado en la sección anterior, los patrones con seem que hemos encontrado en nuestro corpus son seem to, copular seem, it seems (that) y it seems as if. De ellos, la forma seem to es con mucho el mecanismo más usado en los textos origen, seguido de seem copulativo, las matrices it seems (that) y it seems as if. Mostramos en el Gráfico 1 los resultados de forma comparada para que se puedan contrastar los casos encontrados. 


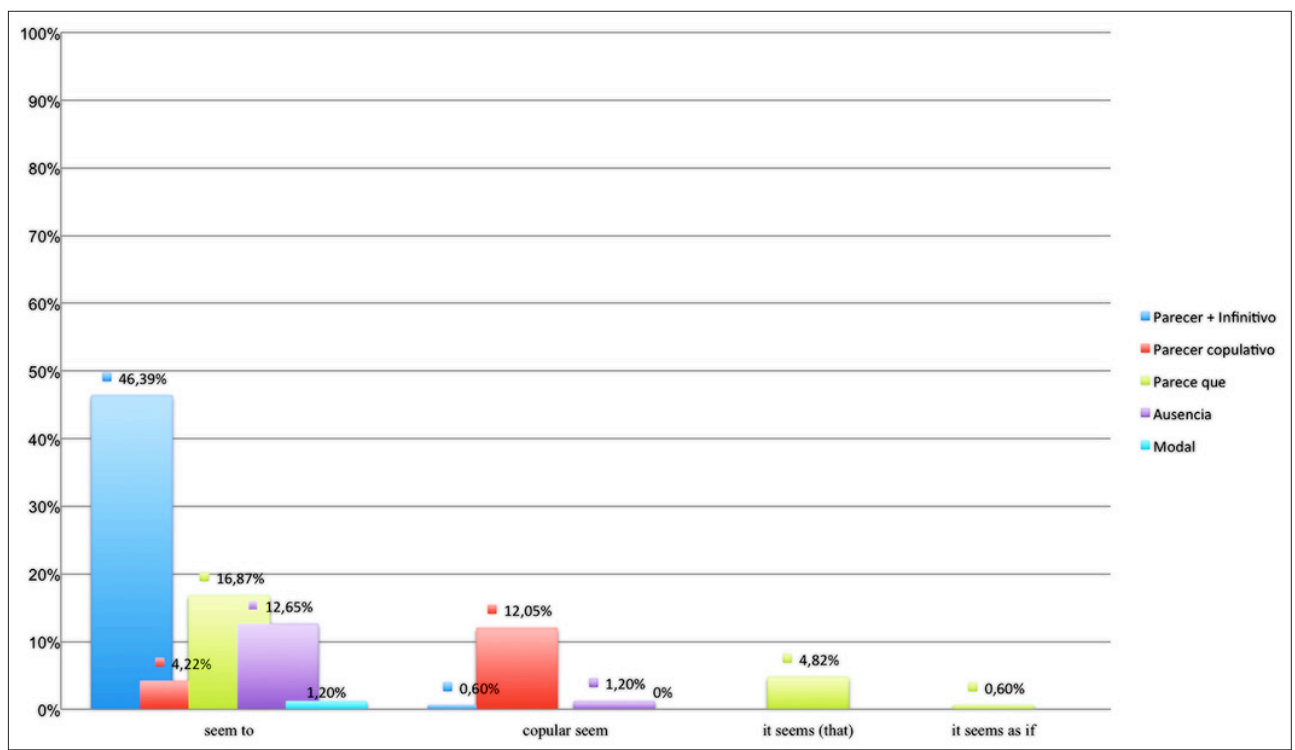

Gráfico 1. Valores de seem en el texto meta (porcentajes).

Como podemos apreciar, la forma más utilizada en el corpus analizado es la traducción de seem to a parecer + infinitivo en español. La traducción de seem como modal es la menos frecuente con solo 2 casos. A continuación comentaremos las distintas acepciones del verbo seem y los ejemplos que hemos encontrado en nuestro análisis.

\subsection{Seem to}

La estructura seem to en los textos origen es la más frecuente, un 81,33\% (135 casos). Esta estructura presenta mucha variación con respecto a su traducción en los textos meta, como se observa en los ejemplos (10)-(15), que mostramos a continuación, donde se destacan las estructuras comparadas en negrita:

(10) The folder to put your database in does not seem to exist: \%1 Would you like digiKam to create it for you? (A 52-KD1).

La carpeta para poner su base de datos no existe: \% ¿Desea que la cree digiKam?

(11) The selected ICC workspace profile path seems to be invalid. Please check it (AB 49-KD1).

La ruta del perfil ICC del espacio de trabajo no parece válida. Por favor, compruébela.

(12) KPhoto Album does not seem to be build with support for all its features. The following is a list indicating to you what you may miss: \%1 For details on how to solve this problem, please choose Help_BAR_KPhoto_Album Feature Status from the menus (AC 56-KD1). 
Parece que KPhotoAlbum no está compilado con todas sus funciones. En la lista siguiente se detallan las que faltan: \%1. Si quiere obtener detalles para solucionar este problema, seleccione Ayuda_BAR_Estado de las características de KPhotoAlbum en el menú.

(13) There seems to be an error in the information entered (\%1), please try again (AI 58KD1).

Hay un error en la información introducida ( \%1), pruebe de nuevo.

(14) Certain characters or punctuation seem to be producing errors in the synthesizer, making it skip entire sentences, or pronounce gibberish. What can I do? (AY 14-KD1).

Algunos caracteres de puntuación pueden producir errores en el sintetizador, haciendo que se salte frases completas, o que pronuncie un verdadero galimatías. ¿Qué puedo hacer?

(15) Kefontinst and kfontview should probably get a mention too, since they don't seem to have any existing docs (20KD-1).

kfontinst y kfontview también merecen ser mencionados, pero no parece haber documentación.

La transposición de esta formal verbal en español es 'parecer + infinitivo' en más del $46 \%$ de los casos, en concreto 77, como se aprecia en la Tabla 1. Mostramos un ejemplo de este caso en (15), seguido de la matriz 'parece que', que enmarca la proposición en español, como vemos en el ejemplo (12). Más de un $12 \%$ de las veces (22 casos) presenta una interpretación de la forma seem como indicador de evento real, lo que se representa con el uso de una forma en presente. Esto lo mostramos en los ejemplos (10) y (13) lo que tiene gran importancia para la categorización de seem como hedge en el sentido que apunta Hyland (1998) y que se ha descrito anteriormente, pues demuestra que la interpretación de este verbo con valor epistémico no siempre puede apoyarse en el contexto lingüístico, ni en el ambiental (Sperber \& Wilson, 1986). En estos dos ejemplos, el uso de las formas de presente en español, 'existe' y 'hay' para las expresiones inglesas seem to exist y seem to be en (10) y (13), respectivamente, se usan porque el contexto así lo admite. El análisis de las evidencias permite el empleo de seem en los textos origen, por lo que esta forma verbal manifiesta el resultado de un razonamiento lógico. En las variantes de los textos meta, el traductor, al omitir la forma seem, prescinde de la posibilidad de indicar la manera en la que los autores han obtenido su información.

Existen otros ejemplos donde ocurre la ausencia de seem en la traducción, como en los ejemplos (16) y (17), que mostramos a continuación: 
(16) Just choose one of the entries which seems to match the style of the CD you rip. It's not important for this example, we don't submit the $C D$ data anyways. For more info go to freedb.org and read about the categories. Pop or Rap for instance are listed under Rock (6-KD1).

Seleccione la entrada que mejor encaje con el estilo del CD que desea copiar. De todos modos, no es importante para este ejemplo, ya que no enviaremos los datos del CD. Para obtener más información vaya a freedb.org e infórmese acerca de las categorías. Por ejemplo, el pop y el rap están bajo la categoría rock.

(17) You do not seem to have writtlen access to this database folder. Without this access, the caption and tag features will not work (50-KD1).

No tiene permisos de escritura en esta ruta. Sin este permiso, las funcionalidades de comentarios y etiquetas no funcionarán.

Mientras que (17) responde al patrón descrito anteriormente donde el grado de factualidad se logra mediante el uso de una forma verbal en presente, esto es 'tiene', en (16), seems to match se reinterpreta como 'mejor encaje'. La selección del adverbio 'mejor' en el texto meta mantiene el valor evidencial de la forma en el texto origen pues alude exclusivamente a la entrada que ofrece evidencias de encajar con el tipo de $\mathrm{CD}$ del que se habla en el ejemplo. De ninguna manera se trata aquí de probabilidad o de posibilidad, pues se entiende por el contexto que una de las entradas encajará con el estilo del CD, de ahí que seem to refleje una conclusión que se desprende de las evidencias que se poseen. Volviendo al ejemplo (17), el uso del adverbio not afecta la interpretación del verbo seem que forma parte de la matriz que introduce la subordinada have written access to this database. Así, la traducción de you do not seem to have written access por la aserción 'no tiene permisos de escritura' indica que el traductor ha valorado you do not seem to como una expresión evidencial que indica el grado de implicación del autor en la construcción del conocimiento (Cornillie \& Delbecque, 2008) pues se indica que have written access se deduce a partir del análisis de las evidencias y que la traducción muestra con el fáctico 'no tiene'. En este sentido, se descarta el uso epistémico de seem en la traducción pero también se elimina todo rastro de la implicación del autor en la configuración del mensaje en el texto meta.

Con menor frecuencia, 7 casos $(4,22 \%)$, seem to se interpreta con valor copulativo tal y como se muestra en el ejemplo (11) y, curiosamente, podemos ver que se traduce con la forma modal 'poder' que puede significar valor dinámico (Collins, 2009) o epistémico. En este sentido, la traducción de 'Certain characters or punctuation seem to be producing...' es: 'Algunos caracteres de puntuación pueden producir', lo que indica que el traductor ha interpretado la conclusión a la que ha llegado el autor del texto original (TO), esto es, el valor evidencial, y que reproduce en español como un hecho mediante el uso del modal dinámico 'poder'. Este uso del modal dinámico significaría que se reconoce el potencial al error como característica intrínseca de los caracteres. Cabe 
también la idea de que el autor del TO interprete seem con valor epistémico, indicando la falta de seguridad de que el error que producen los caracteres tenga lugar en el futuro. Este valor quedaría perfectamente representado con 'poder'. Desde nuestro punto de vista, pensamos que la primera opción sería la más adecuada por lo que el conjunto seem to be producing vendría a indicar una conclusión a partir de evidencias. Pietrandrea y Stathi (2010: 346), en su estudio sobre modalidad, determinan al respecto lo siguiente:

"It could be claimed, therefore, that the evidential meaning is not expressed by the modal verb per se, rather by complex periphrastic constructions combining the modal verb, the infinitive/dependent clause and specific aspectual values of the dependent verb".

Esto es aplicable a nuestro ejemplo (11) de seem que adquiere su significado evidencial mediante la combinación con el aspecto progresivo del verbo 'produce'.

\subsection{Seem copulativo}

La forma seem con valor copulativo en oraciones como las que se incluyen en los ejemplos del (18) al (20) aparecen con una frecuencia del 13,85\% (23 casos).

(18) There is one more expression that may seem odd ifyou haven 't seen it in other languages, the ternary conditional operator (B 167-PHP).

Hay otra expresión que puede parecer extraña si no la has visto en otros lengua es, el operador condicional ternario.

(19) APS 'pts seems out of order (actual pts \%1, last seen pts \%2) (BA 63-KD1).

Los pts de APS parecen estar fuera de servicio (pts real \%, último pts observado $\% 2)$.

(20) If the last two examples above seem odd, see String conversion to numbers (BI 168-PHP).

Si se desea obligar a que una variable sea evaluada con un tipo concreto, mire la sección. Forzado de tipos.

Las traducciones identifican esta forma con los valores copulativos en un 12,05\% (20 casos), como podemos ver en el ejemplo (18) donde, prácticamente, se ha llevado a cabo una transposición de los constituyentes sintácticos del texto original al texto meta. Tanto en el caso de seem como en el de 'parecer', se trata de verbos copulativos de percepción, pero que, lejos de manifestar fuente o modo de información, su uso se asienta en una comparación basada en la evaluación subjetiva de one more expression, según apunta Cornillie (2012) siguiendo a Bolinger (1991). El autor identifica la expresión como odd (extraña), dada la condición que introduce if. Esta evaluación viene determinada lógicamente por la comparación con otras expresiones que no le son extrañas al autor. 
En el ejemplo (19), el traductor interpreta el seem copulativo como 'parecen' seguido del infinitivo 'estar'. Este último verbo captura el valor copulativo de seem en el texto origen. El uso de 'parece' en este caso puede ser sustituido por la expresión 'da la impresión de que', que indica la manera en la que se ha obtenido la información. Finalmente, en el ejemplo (20), se opta por un verbo en presente diferente a 'parecer', con cierto cambio del significado en el texto meta. En este caso, la traducción de seem parece responder a un conocimiento externo que el traductor tiene sobre la materia de la que se habla, pues no parece que haya una correspondencia lógica entre If the last two examples above seem odd y su traducción al español 'Si se desea obligar a que una variable sea evaluada con un tipo concreto'.

En ninguno de los casos que se han analizado en esta sección, el verbo seem manifiesta duda o, por lo menos, los contextos en los que esta forma copulativa aparece no permiten concluir que tenga un significado epistémico definido. Sin embargo, uno de los casos indica la interpretación de seem copulativo como un mecanismo evidencial.

\subsection{It seems (that)}

La matriz it seems (that) no aparece con frecuencia en nuestro corpus, tan solo un $4,82 \%$ ( 7 casos), lo que indica la posibilidad de que esta estructura se encuentre en desuso en la lengua inglesa de especialidad, pero esta hipótesis solo la podríamos determinar con el análisis de un corpus de trabajo más amplio. Las forma registrada en el sub-corpus español para esta estructura es 'parece que', como se muestra abajo en los ejemplos (21) y (22). En estos dos casos, se mantiene la matriz evidencial para expresar que la información que se ofrece se ha obtenido mediante el análisis de la situación a la luz de los indicios de los que se dispone.

(21) It seems that the folder \%1 was deleted. Do you want to delete it from the server? (C 125-KD2)

Parece que se eliminó la carpeta \%1 ¿ ¿Quiere eliminarla del servidor?

(22) It seems powersaved is running on this system. PowerDevil will not start if other power managers are active. If you want to use PowerDevil as your primary power manager, please stop powersaved and restart the PowerDevil service (CA 81-KD1).

Parece que se está ejecutando powersaved en este sistema. PowerDevil no arrancará si hay otro gestor de energía activo. Si quiere usar PowerDevil como su gestor primario de energía, pare powersaved y rearranque el servicio de PowerDevil.

El valor evidencial y la ausencia de valor epistémico de seem se observan claramente en el ejemplo (23):

(23) It may seem that amarok has the same old features found in the average audioplayer, but we did not stop here (3-KD1). 
Puede parecer que amarok tiene las mismas características que se pueden encontrar en un reproductor de audio de gama media, pero no se detiene aquí.

Tanto en el texto original como en el meta se mantiene la misma estructura evidencial con seem para indicar la fuente de donde se ha obtenido la proposición que sigue a esta matriz. El componente epistémico lo aporta el uso de la forma modal may. De aquí se desprende que, si seem tuviese un valor epistémico en cualquiera de las dos lenguas, no se podría entender la combinación may + seem, pues estaríamos ante una redundancia funcional. Es evidente que may es una estrategia epistémica para expresar un grado indeterminado de probabilidad de que la conclusión a la que se llega sea cierta.

\subsection{It seems as if}

La estructura it seems as if solo aparece en una ocasión en todo el corpus que hemos analizado y se presenta en el ejemplo (24), a continuación:

(24) The Correct for atmospheric refraction checkbox controls whether the positions of objects are corrected for the effects of the atmosphere. Because the atmosphere is a spherical shell, light from outer space is bent as it passes through the atmosphere to our telescopes or eyes on the Earth's surface. The effect is largest for objects near the horizon, and actually changes the predicted rise or set times of objects by a few minutes. In fact, when you see a sunset, the Sun's actual position is already well below the horizon; atmospheric refraction makes it seem as if the Sun is still in the sky. Note that atmospheric refraction is never applied if you are using Equatorial coordinates (DC 25-KD1).

La casilla Corrección para la refracción atmosférica controla si las posiciones de los objetos se deben corregir debido a los efectos de la atmósfera. Como la atmósfera es una envoltura esférica, la luz del espacio exterior se 'dobla' cuando pasa a través de la atmósfera antes de llegar al telescopio o a nuestros ojos, situados sobre la superficie de la Tierra. Este efecto es mayor para los objetos que están cerca del horizonte y afecta realmente a las horas de orto y ocaso de estos objetos en varios minutos. De hecho, cuando usted 've' un ocaso, la posición real del Sol ya está muy por debajo del horizonte; la refracción atmosférica hace que parezca que el Sol está aún en el cielo. Tenga en cuenta que la refracción atmosférica no se aplicará nunca cuando esté usando coordenadas ecuatoriales.

En español, se elimina el elemento que expresa la comparación, esto es, as if, $\mathrm{y}$ se opta por la estructura 'parecer que'. Tal y como se apunta en Alonso Almeida y Lareo (en prensa), la estructura comparativa as if marca claramente "the result of the author's inferential processes" de las evidencias analizadas, además de "invoking some kind of uncertainty", aunque estos autores apuntan que no se puede asegurar esta idea de incertidumbre puesto que, por lo general, el uso de as if demuestra el razonamiento lógico mediante la utilización de las premisas usadas en el supuesto lógico. Se prescinde de la expresión de este tipo de razonamiento en el texto meta al 
no usar la partícula as if. Sin embargo, el uso de as if funciona como un subjetivizador, indicando claramente el punto de vista del autor. En este contexto, seem as if indica que la información que aparece en la cláusula subordinada no es cierta, aunque las evidencias apunten a ello.

Gisborne (2010) afirma que el significado de las expresiones que él denomina LIKE/C y que incluyen verbos de percepción seguidos de like + 'cláusula' o de as if/ though + 'cláusula' tienen el significado de resemble aunque apunta que "this notion of resembling can become rather abstract". En el caso del ejemplo (24), el razonamiento lógico es el adecuado, pero el resultado no se corresponde con la realidad del evento que se pretende describir. La duda está en saber hasta qué punto este uso de seem puede considerarse como epistémico pues no se aprecia ningún atisbo de duda acerca de la certeza del hecho, si bien la conclusión o la deducción del proceso inferencial que se describe no es cierto y esto se señala inequívocamente por as if. En el análisis de un ejemplo similar, Nakamura (2013: 2) aprecia el significado epistémico de los verbos de percepción con as if: "Here, look as if(although)/ like function like modal expressions to avoid assertion, similar to adverbs such as maybe".

\section{CONCLUSIÓN}

El estudio realizado sobre un corpus paralelo de textos técnicos demuestra que, por lo general, la traducción de seem en español tiende a reflejar la forma sintáctica de la lengua original. En cuanto al valor semántico, éste varía en ocasiones según la interpretación que el traductor haga del texto origen. En este sentido, hemos atestiguado que la forma evidencial seem to se traduce tanto por 'parecer' seguido de infinitivo, o bien mediante matrices evidenciales. Cabe destacar que esta estructura seem to, al igual que otras de igual significado, se presenta tanto con verbos modales como con verbos de significado factual en la lengua meta. En cuanto a seem con valor copulativo, no se observa una tendencia conservadora en todos los casos y aparece en el texto meta también como evidencial.

La estructura seem as if, cuya presencia en el corpus se reduce a un único ejemplo, se usa para expresar un razonamiento que da lugar a una información que puede ser, en cierta manera, figurada o bien el resultado de una comparación. Por este motivo, seem as if puede entenderse como un hedge en tanto que se trata de atenuar la afirmación que se comunica. Sin embargo, hemos apuntado que, realmente, este mecanismo no parece indicar falta de seguridad del hablante, pues la proposición surge a raíz de una evaluación de las premisas disponibles. Es por eso que lo hemos categorizado como un mecanismo evidencial.

Todo lo expuesto invita a una reflexión sobre la categorización de seem como verbo epistémico que puede indicar probabilidad o falta de seguridad del hablante, tal y como se apunta en Mauranen (1997), Wierzbicka (2006) y Aijmer (2009). Los datos analizados sugieren que, lejos de expresar significado epistémico, seem refleja el modo 
de adquisición de la información indicando un proceso cognitivo basado puramente en evidencias. En cuanto al uso copulativo de esta forma, no expresa valor evidencial y su empleo procede de un proceso de comparación con respecto a otro sujeto o evento.

En trabajos futuros, estudiaremos otros verbos de percepción relacionados con el verbo seem, como por ejemplo look, desde una perspectiva contrastiva como hemos hecho aquí. Nuestro propósito es determinar el significado contextual de estos verbos y su posible clasificación dentro del mapa semántico de la modalidad en lengua inglesa, así como las correspondencias que estos adquieren en español. 


\section{REFERENCIAS BIBLIOGRÁFICAS}

Aijmer, K. (2009). Seem and evidentiality. Functions of Language, 16(1), 63-88.

Aikhenvald, A. (2004). Evidentiality. Oxford: Oxford University Press.

Alonso Almeida, F. (2012). An analysis of hedging in eighteenth century English astronomy texts. En I. Moskowich \& B. Crespo (Eds.), Astronomy "playne and simple": The Writing of Science Between 1700 and 1900 (pp. 199-220). Ámsterdam: John Benjamins.

Alonso Almeida, F. \& Lareo, I. (en prensa). The Status of Seem in Nineteenthcentury CePhit. En I. Moskowich \& B. Crespo (Eds.), 'The conditioned and the unconditioned'. Late Modern English Texts on Pbilosophy. Ámsterdam: John Benjamins.

Beke, R. (2005). El metadiscurso interpersonal en artículos de investigación. Revista Signos. Estudios de Lingïistica, 38(56), 7-18.

Bolinger, D. (1991). Essays on Spanish. Words and Grammar. Newark Delaware: Juan de la Cuesta Hispanic Monographs.

Chafe, W. (1986). Evidentiality in English Conversation and Academic Writing. En W. Chafe \& J. Nichols (Eds.), Evidentiality: The Linguistic Coding of Epistemology (pp. 261-272). Norwood: Ablex.

Collins, P. (2009). Modals and Quasi-modals. Ámsterdam \& Nueva York: Rodopi.

Cornillie, B. (2009). Evidentiality and Epistemic Modality. On the Close Relationship Between Two Different Categories. Functions of Language, 16(1), 44-62.

Cornillie, B. (2012). Sobre la gramaticalización y la (inter)subjetividad de los (semi) auxiliares evidenciales parecer y resultar. Ponencia presentada en la Universidad de Sevilla, España.

Cornillie, B. \& Delbecque, N. (2008). Speaker Commitment: Back to the Speaker. Evidence from Spanish Alternations. Belgian Journal of Linguistics, 22, 37-62.

Dendale, P. \& Tasmowski, L. (2001). Introduction: Evidentiality and Related Notions. Journal of Pragmatics, 33(3), 339-348.

Diewald, G., Kresic, M. \& Smirnova, E. (2009). The Grammaticalization Channels of Evidentials and Modal Particles in German: Integration in Textual Structures as a Common Feature. En M. M. Hansen \& J. Visconti (Eds.), Current Trends in Diachronic Semantics and Pragmatics (pp. 189-210). Londres: Emerald. 
Ferrari, L. (2006). Evaluación y modalidad en artículos de investigación provenientes de diferentes disciplinas. En D. Kaiser, K. Jungbluth \& G. E. Ciapuscio (Eds.), Sincronía y diacronia de tradiciones discursivas en Latinoamérica (pp. 27-38). Vervuert: Biblioteca Ibero Americana.

Gisborne, N. (2010). The Event Structure of Perception Verbs. Oxford: Oxford University Press.

Hyland, K. (1998). Hedging in Scientific Research Articles. Ámsterdam: John Benjamins.

Johansson, S. (2007). The English Verb Seem and Its Correspondences in Norwegian. What Seems to Be the Problem? En S. Johansson (Ed.), Seeing Through Multilingual Corpora: on the Use of Corpora in Contrastive Studies (pp. 117-138). Ámsterdam: John Benjamins.

Jonson, B. (1983). El alquimista. Barcelona: Icaria Editorial.

Kranich, S. (2009). Epistemic Modality in English Popular Scientific Texts and their German Translations. Trans-kom, 2(1), 26-41.

Marín-Arrese, J. (2009). Effective vs. Epistemic Stance, and Subjectivity/ Intersubjectivity in Political Discourse. A Case Study. En A. Tsangalidis \& R. Facchinetti (Eds.), Studies on English Modality. In Honour of Frank R. Palmer. Linguistic Insights. Studies in Language and Communication 111 (pp. 23-52). Bern: Peter Lang.

Mauranen, A. (1997). Hedging in Language Revisers Hands. En R. Markkanen \& H. Schröder (Eds.), Hedging and Discourse: Approaches to the Analysis of a Pragmatic Phenomenon in Academic Texts (pp. 115-133). Berlín \& Nueva York: Walter de Gruyter.

Mendiluce Cabrera, G. \& Hernández Bartolomé, A. I. (2010). La matización asertiva en el artículo biomédico: Una propuesta de clasificación para los estudios contrastivos inglés-español. Ibérica, 10, 63-90.

Morales, O. \& Cassany, D. (2008). Interpretaciones de la atenuación en artículos de revisión de Odontología. Revista Signos. Estudios de Lingüística, 41(67), 299-328.

Morales, O. A., Comas, D. C., Olmo, S. O. D., Peña, C. G. \& Altuve, E. I. M. (2009). ¿Es la escritura académica odontológica hispanoamericana un discurso matizado?: Estudio de la atenuación en artículos de investigación. LFE: Revista de Lenguas para Fines Especificos 15, 135-157.

Mushin, I. (2001). Evidentiality and epistemological stance: Narrative retelling. Ámsterdam: John Benjamins. 
Nakamura, F. (2010). Uncovering of rare or unknown usages: A history of seem meaning to pretend. En M. Kytö (Ed.), Language Change and Variation From Old English to Late Modern English: A Festschrift for Minoji Akimoto (pp. 217-238). Berna: Peter Lang.

Nakamura, F. (2013). The development and (De)subjectification of the English copulative perception verbs with special reference to modern American English. 6th International Spring Forum 2013. The English Linguistic Society of Japan (ELSJ) [en linea]. Disponible en: http://elsj.kaitakusha.co.jp/2013forum/ Fuminori\%20Nakamura.pdf

Nuyts, J. (2001). Subjectivity as an evidential dimension in epistemic modal expressions. Journal of Pragmatics, 33(3), 383-400.

Palmer, F. (1986). Mood and Modality. Londres: Longman.

Pepys, S. (2007). Diario Samuel Pepys 1660-1669. Madrid: Espasa.

Pietrandrea, P. \& Stathi, K. (2010). What counts as an evidential unit? The case of evidential complex constructions in Italian and modern Greek. STUF -Language Typology and Universals, 63(4), 345-357.

Sperber, D. \& Wilson, D. (1986). Relevance: Communication and cognition. Oxford: Basil Blackwell.

Usoniene, A. (2000). On the modality of the English verbs of seeming. Belgian Journal of Linguistics, 14(1), 185-205.

Wierzbicka, A. (2006). English: Meaning and culture. Oxford: Oxford University Press.

Willet, T. (1988). A cross-linguistic survey of the grammaticalization of evidentiality. Studies in Language, 12(1), 51-96.

\section{NOTAS}

1 Este trabajo forma parte del Proyecto de investigación "Modalidad epistémica, evidencialidad y gramaticalidad: Análisis contrastivo inter e intralingǘstico en el discurso oral y escrito" (MODEVIG) (clave FFI2011-25755), financiado por el Ministerio de Ciencia e Innovación (España). Las primeras conclusiones de este trabajo se presentaron en el VI Congreso Internacional de la Asociación Ibérica de Estudios de Traducción e Interpretación (AIETI), celebrado en Las Palmas de Gran Canaria, 23-25 de enero de 2013.

2 En este artículo usamos la vOz inglesa hedge puesto que existen diferentes formas en español para referirse a este fenómeno, todas con sus peculiaridades: matización (Mendiluce Cabrera \& Hernández Bartolomé, 2010), mitigación (Beke, 2005), atenuación (Morales \& Cassany, 2008; Morales, Comas, Olmo, Peña \& Altuve, 2009). 